\title{
CONSECUENCIAS Y RESPONSABILIDADES DE LA EMPRESA ANTE EL MOBBING
}

Consequences and responsibilities of the company before the mobbing

\section{María Concepción Verona Martel $^{*}$ José Juan Déniz Mayor** Raquel Santana Mateo ${ }^{* * *}$}

Resumen: El mobbing o acoso psicológico en el trabajo se ha constituido en un grave problema en el mundo laboral del siglo XXI, ocasionando graves efectos tanto para la persona que lo sufre como para la organización en cuyo seno tiene lugar. Es un error confundirlo con la conflictividad cotidiana que se da en todos los ambientes laborales. Sus efectos en las víctimas son devastadores y también en las organizaciones en las que acontecen. Las empresas no pueden obviar su responsabilidad en este tema $\mathrm{y}$ deben tomar de forma urgente las medidas para prevenirlo $\mathrm{y}$ erradicarlo.

Profesora titular de la Facultad de Economía, Empresa y Turismo, de la Universidad de Las Palmas de Gran Canaria, España. E-mail: cverona@defc.ulpgc.es.

* Profesor titular de la Facultad de Economía, Empresa y Turismo de la Universidad de Las Palmas de Gran Canaria, España. E-mail: josejuan.deniz@ulpgc.es

*** Graduada en Turismo por la Universidad de Las Palmas de Gran Canaria. E-mail: rake115700@hotmail.com 
Los resultados del estudio muestran que la empresa debe velar por sus trabajadores, pues constituyen su capital más valioso, ella es responsable de lo que les acontezca y por tanto debe controlar el mobbing.

Palabras clave: mobbing, empresa, responsabilidad.

Clasificación JEL: J790, J830, M100.

Abstract: Bullying or psychological harassment in the workplace has become a serious problem in the working world of the century, causing serious damage to both the person who suffers it and the organization within which occurs. It is a mistake to confuse it with everyday conflict that occurs in all work environments. Its effects on victims are devastating and in the organizations that happen. Companies cannot ignore its responsibility in this matter and must take urgent measures to prevent and eradicate it.

The results of the study show that the company must ensure their workers, as they are their most valuable asset, she is responsible for what happens to them and therefore must control the mobbing.

Keywords: mobbing, firm, responsibility.

JEL classification: J790, J830, M100. 


\section{Introducción}

El mobbing tiene lugar en una organización y por tanto dicha organización es responsable de este grave problema laboral, por tal motivo, el objetivo del presente artículo es poner de manifiesto las consecuencias y responsabilidades de la organización ante el mobbing.

El mobbing o acoso psicológico en el trabajo se ha constituido en un grave problema en el mundo laboral del siglo XXI, ocasionando graves efectos tanto para la persona que lo sufre como para la organización en cuyo seno tiene lugar. Es un error confundirlo con la conflictividad cotidiana que se da en todos los ambientes laborales debido a las tensiones y a la convivencia forzada en espacios restringidos (Sánchez, 1999: 238); el mobbing es mucho más que eso. Según el Diccionario Panhispánico de Dudas (2005), el término mobbing es una

Voz inglesa con que se designa el hostigamiento al que, de forma sistemática, se ve sometida una persona en el ámbito laboral, y que suele provocarle serios trastornos psicológicos. ${ }^{1}$

El mobbing no es un acto puntual o aislado, una impertinencia dicha una vez es y sigue siendo una impertinencia, pero como afirma Leymann (1996a: 27), si se repite cada día, durante semanas, entonces cabe hablar de mobbing. El acoso psicológico en el trabajo supone el desconocimiento o desprecio de la dignidad de la víctima, la cual ve rebajada la consideración que merece no solo como profesional sino también como persona.

Leymann (1996a: 27, 40-41) considera que el mobbing implica comportamientos o comunicaciones hostiles e inmorales que son dirigidas de forma sistemática y recurrente por uno o varios individuos con la finalidad de destruir sus redes de comunicación, su

1 Si bien el diccionario propone que en los textos en español dicho vocablo se sustituya por el equivalente acoso laboral, en el presente artículo se utilizará la expresión inglesa por ser la más usual en los estudios sobre la materia. 
reputación, o perturbar el ejercicio de su trabajo y lograr finalmente que abandone el lugar de trabajo; estas acciones se producen con una frecuencia de, al menos, una vez por semana y con una duración mínima de seis meses.

El motivo por el que un acosador inicia el proceso de mobbing es difícil de determinar; algunos autores piensan que se inicia como consecuencia de un conflicto profesional mal resuelto (Leymann, 1996a), debido a que no se ha conseguido establecer un conflicto (Hirigoyen, 2001), o más directamente porque se desea despedir a un trabajador y no se ha encontrado un argumento fundamentado para hacerlo (López y Vázquez, 2003). Pero tal vez el motivo por el que una persona ocasiona a otra un daño tan grave como el asociado al mobbing sea mucho más simple: el acosador "ha descubierto" a una persona que posee unas características que no puede soportar y toma la decisión de eliminarla sin dejar huellas. Lo más significativo de este hecho es que tales características son las que generalmente en una sociedad sana se utilizan para calificar a alguien como "buena persona".

La Fundación Europea para la mejora de las Condiciones de Vida y de Trabajo (EUROFOUND) ${ }^{2}$ realizó en el año 2005 la cuarta encuesta europea ${ }^{3}$ sobre las condiciones del trabajo considerando la información de 31 países europeos. Los resultados obtenidos mostraban que uno de cada 20 trabajadores (5\%) ha padecido el acoso psicológico en el trabajo ( $4 \%$ de hombres y $6 \%$ de mujeres). La quinta encuesta realizada en el año 2010, basada en datos de

2 EUROFOUND es un organismo tripartito de la Unión Europea creado en 1975 para contribuir a la planificación y creación de mejores condiciones de vida y de trabajo en Europa. De manera más concreta, evalúa y analiza las condiciones de vida y de trabajo, emite dictámenes autorizados y asesora a los responsables y principales actores de la política social, contribuye a la mejora de la calidad de vida e informa sobre la evolución y las tendencias en este ámbito, en particular, sobre los factores de cambio.

3 La primera encuesta correspondiente al período 1990-1991 ni siquiera consideraba esta cuestión. Estos estudios se realizan cada 5 años. 
34 países europeos ${ }^{4}$, destaca que muchos países han incrementado el porcentaje ${ }^{5}$ de afectados. Ya en la cuarta encuesta se decía que el mobbing iba en aumento con el paso de los años. Además se sigue observando que, por lo general, este problema afecta más a las mujeres que a los hombres.

Parece que el mobbing es una actividad humana común, practicada en todos los países y en la mayoría de las organizaciones (Vega y Comer, 2005: 108). Leymann (1996a: 216-217) señala que el mobbing no se puede justificar moralmente basándose en el hecho de que es frecuente, aunque solo sea porque va en contra de los fundamentos morales de las sociedades occidentales.

Lo cierto es que el mobbing no tiene justificación posible, destruir a una persona por el simple capricho no es admisible en ninguna sociedad sana. Ante esto, tal como afirma Girard (2002: 84), "para favorecer la violencia colectiva, hay que reforzar su inconsciencia (...). Y, al contrario, para desalentar esa violencia, hay que mostrarla a plena luz, hay que desenmascararla".

El trabajo se divide en las siguientes secciones: tras la introducción, la sección dos recoge el perfil de la empresa fomentadora del mobbing, los sectores económicos más afectados por este tipo de acoso se detallan en la sección tercera, la cuarta sección hace referencia a las consecuencias y responsabilidades de la empresa en cuyo seno existe el mobbing, quedando la última sección para la presentación de las conclusiones.

4 La quinta encuesta no presenta sus resultados tan detalladamente como lo hace la cuarta, de ahí que en muchas ocasiones se haga mayor referencia a los datos de la cuarta.

5 En la quinta encuesta de la EUROFOUND se menciona que la diferencia de porcentajes de mobbing entre países podría ser, tal vez, debida no al hecho de que no exista este problema en el país sino como consecuencia del efecto de diferencias culturales que pueden provocar que un mismo comportamiento sea considerado mobbing en un país y en otro no, o bien, que sabiendo la víctima que está siendo objeto de mobbing considere que socialmente no es aceptable hacerlo público. Tanto una cuestión como otra es desde un punto de vista humano inaceptable y sólo provoca que los acosadores proliferen por doquier. 


\section{Perfil de la empresa favorecedora del mobbing}

Así como puede elaborarse un perfil de las partes implicadas en el proceso de mobbing (víctima, acosador y cómplices), también puede establecerse un perfil de la empresa que favorece que se produzcan en ella casos de acoso psicológico en el trabajo.

El carácter cerrado o autosuficiente de algunas organizaciones y que en ellas prevalezcan aspectos como el poder o el control sobre la productividad, la cooperación o la eficacia, unido al miedo del trabajador al desempleo (aspecto que se vuelve mucho más crítico en momentos de crisis económica) son factores clave para que en una empresa aparezca el mobbing (López y Vázquez, 2003: 75).

A lo anterior se pueden añadir otros aspectos que también favorecen la aparición del acoso psicológico en el trabajo en una empresa como son (López y Vázquez, 2003; Muñoz et al., 2006):

a) Una pobre organización del trabajo.

b) Una deficiente gestión de los conflictos.

c) Un estilo de dirección autoritario.

d) El fomento de la competitividad interpersonal como valor cultural.

e) La ausencia de ética empresarial.

f) La falta de formación en management y liderazgo.

g) El fomento del clima de inseguridad personal y laboral.

h) La persecución de la solidaridad, la confianza y el compañerismo como valores sospechosos o antiempresariales.

i) Una alta burocratización.

j) Una elevada sobrecarga de trabajo.

k) Que la productividad no sea evaluada de forma externa. 
Moreno et al. (2005: 630) muestran en su estudio empírico como una pobre política organizacional y la existencia de contratos temporales o de obra (inestabilidad laboral) están asociados con la aparición del acoso psicológico en el trabajo. En el estudio empírico realizado por Muñoz et al. (2006: 355) se pone de manifiesto que en las organizaciones el clima de apoyo y el clima de reglas se relacionan con una menor presencia de prácticas de acoso psicológico, mientras que el clima de metas parece ser un entorno propicio para las prácticas de mobbing al igual que los procesos de cambio organizacional. Por su parte, Rodríguez et al. (2006: 333) demuestran en su estudio empírico que la dificultad de la tarea a realizar y un exigente ritmo de trabajo favorecen la aparición del mobbing, mientras que la autonomía y reconocimiento del trabajo realizado atenúan su presencia en la organización. Por su parte, Topa et al. (2006: 766) comprueban empíricamente que la cultura que exista en la organización fomentará o desalentará la aparición del mobbing, ocurriendo lo primero si se permite o alienta la violencia psíquica y lo segundo si no es tolerada.

En este apartado tiene cabida hacer referencia al neomanagement, esa forma de dirigir organizaciones y personas mediante la continua destrucción de los recursos humanos, del clima laboral y del entorno organizativo. Considerado como el perfecto caldo de cultivo para la aparición del estrés, del burnout y del mobbing, entre otros, cuenta con dos grandes protagonistas: los jefes tóxicos y sus víctimas, lo que permite hablar de la existencia de "organizaciones tóxicas" en el mundo empresarial, pues trabajar en ellas perjudica la salud de una gran parte de sus trabajadores (Piñuel, 2004: 69). El neomanagement es definido por Piñuel (2004: 24) como:

Un conjunto de enunciados y prescripciones en materia de dirección de empresas y personas que se fundamenta en la ideologia y las "bases" filosófico-religiosas de la nueva economía sacrificial que domina en la actualidad el panorama ideológico empresarial y organizativo, y que son ampliamente compartidos por directivos y trabajadores. 
Este ámbito laboral favorecerá a aquellos individuos más expertos en instrumentalizar y manipular a los demás para sus propios fines. La psicología califica a tales individuos como psicópatas, en este caso concreto al referirnos a organizaciones, se les puede denominar psicópatas organizacionales. Tales individuos son recompensados, promocionados y nombrados directivos estrella dentro de las organizaciones que dirigen, creando a su alrededor un entorno laboral tóxico para los demás trabajadores, a los que les quedan dos opciones: imitar a sus jefes o marcharse de la organización no sin antes haber sufrido considerablemente (Piñuel, 2004: 39). En estos entornos laborales la violencia se hace cada vez más sutil hasta convertirse en una violencia psicológica difícil de acreditar ante los tribunales (2004: 48).

La naturaleza mimética del ser humano y la propia inseguridad de las personas que buscan una trascendencia en la imitación de aquellos que son tenidos como modelos sociales (otros directivos de notorio éxito), explican por qué, a lo largo del tiempo, van a surgir nuevos neomanagers a fuerza de clonar los modelos observados y tenidos por buenos entre los directivos anteriores (Piñuel, 2004: 49-50).

Los psicópatas organizacionales aniquilan el capital intelectual humano y extinguen la creatividad, la iniciativa y la comunicación en las organizaciones en las que se instalan, ello da lugar a que las personas subyugadas o sometidas por el miedo se transformen en trabajadores desmotivados y alienados de su propio trabajo y así, propagan a su alrededor una atmósfera de mediocridad y de temor. Pero no todas las empresas son iguales y algunas, no muchas, se dan cuenta de que no resulta de interés para ellas mantener en su seno managers tóxicos dado el coste humano y económico derivado de la devastación organizativa que generalizan a su alrededor (Piñuel, 2004: 77, 103). Desafortunadamente, no todas las empresas piensan así y mantienen y fomentan este tipo de jefes tóxicos, y el hecho de que las cifras de casos de acoso psicológico en el trabajo sigan aumentando es una muestra palpable de ello. 


\section{Sectores económicos más afectados por el mobbing}

Cualquier sector económico puede verse afectado por el mobbing, aunque hay determinados sectores que resultan más afectados que otros. Así, en el sector público predomina el mobbing con más frecuencia que en el sector privado (Hirigoyen, 2001: 110), la razón de ello se relaciona con su carácter más intensamente reglamentado, la obsolescencia de buena parte de sus paradigmas de regulación organizativa, el reglamentismo utilizado y una cultura basada en el control, la jerarquía y el poder (López y Vázquez, 2003: 75).

En cuanto a la duración del proceso de mobbing, este presenta diferencias entre el sector público y el sector privado. Así, mientras que el primero puede durar años en el segundo raramente dura más de un año (Hirigoyen, 2001: 104). Esta diferencia temporal en la duración del acoso psicológico en el trabajo puede ser explicada debido a que en el sector público, en principio, los trabajadores no pueden ser despedidos ni suelen ellos irse por iniciativa propia, se recurre a otro mecanismo para conseguir que abandonen la organización: "destruirlos" mediante el mobbing, en cambio, en el sector privado es más fácil despedir a alguien aunque la ley exige que haya motivos para ello. A ello hay que añadir un aspecto muy relevante y es el hecho de que en el sector privado, al empresario le sale costoso tener a una persona en la empresa sin asignarle ningún trabajo, o bien, realizando tareas inútiles para la firma, en cambio al acosador público le es indiferente esta cuestión porque los sueldos los paga el Estado, no él de su bolsillo. ${ }^{6}$

6 El acosador público está destruyendo psicológicamente a una persona, siendo altamente probable que salga inmune del delito y, además, no tiene en cuenta que lo está haciendo con dinero del Estado. Entonces, ¿si se le fuera a acusar ante la justicia por cuántos delitos se le acusaría? ¿tendría que indemnizar sólo a la víctima de acoso psicológico en el trabajo? 
Las grandes empresas no son las únicas en las que se produce el mobbing, en las pequeñas y medianas compañías también aparece este comportamiento que puede rayar en el sadismo (Hirigoyen, 2001: 126).

El mobbing se encuentra sólidamente instalado en la administración del Estado, en la sanidad, en las universidades, en instituciones altamente reglamentadas y homogéneas (escuelas, Fuerzas Armadas, etc.) y en las instituciones conservadoras que presentan una escasa tolerancia a la diversidad y fuertes vínculos e identidades compartidas entre sus miembros (López y Vázquez, 2003: 75). Más concretamente, si se tiene en cuenta el grado de intensidad del mobbing resulta que las instituciones públicas, las universidades, los hospitales, los centros de enseñanza, el ejército, el mundo profesional de la investigación, del deporte, de la política (donde ocupan los puestos más elevados). Pero también hay que considerar otros sectores con importante incidencia del acoso psicológico en el trabajo como el sector de la hostelería, los negocios familiares, las instituciones y organizaciones caritativas y las empresas con trabajadores contratados en condiciones precarias (Hirigoyen, 2001; López y Vázquez, 2003).

La cuarta encuesta europea sobre las condiciones del trabajo realizada por EUROFOUND constató que el sector público sufre una mayor incidencia del acoso psicológico en el trabajo $(6 \%)$ que el sector privado (4\%); siendo los sectores más afectados estos: administración pública, educación, sanidad y trabajo social, defensa, hoteles y restaurantes. En cuanto al tamaño de las empresas, las empresas grandes ( + de 250 trabajadores) presentan un mayor índice de acoso que las empresas pequeñas, llegando a elevarse a un $8 \%$. Los resultados de la quinta encuesta están en la misma línea. 


\section{Consecuencias y responsabilidades de la empresa derivadas de la existencia de mobbing en su seno}

\subsection{CONSECUENCIAS PARA LA EMPRESA}

Hay quienes sostienen que en una organización es normal e incluso hasta deseable que se produzca el mobbing, pues consideran que constituye un buen instrumento para crear un entorno de competencia entre los trabajadores permitiendo además seleccionar a los mejores para luchar en un mundo cada vez más complejo e inhumano. Este razonamiento es total y absolutamente calificable de patológico, va en sentido contrario a la ética, a los derechos humanos, a una buena y saludable dirección de la empresa; en definitiva, no tiene cabida en una organización sana, pero sí en una organización enferma, tóxica y perversa.

Las consecuencias perjudiciales de que exista mobbing en una empresa no son únicamente para la persona que lo padece, la víctima, sino que también afecta y, desfavorablemente, a la empresa en la que se produce este deplorable problema. El mobbing no es un juego patológico con resultado cero en el que el fracaso de uno permite la victoria de otro, al contrario, con el mobbing pierden todos: la víctima, en primer lugar, pero también la empresa pues el clima de tensión que ocasiona el mobbing lleva a una caída generalizada en el rendimiento (Sánchez, 1999: 247), y muchos otros efectos igualmente devastadores.

No hay duda de que en las empresas en que se desencadenan los procesos de mobbing experimentan, entre otros efectos (López y Vázquez, 2003):

a) Una disminución de su productividad y de su eficacia por el mal clima de trabajo.

b) Un aumento del tiempo de trabajo perdido en bajas laborales, enfermedades, accidentes e incapacidades. 
c) La necesidad de formar nuevos trabajadores para sustituir a los que están de baja médica o que se han ido, a esto hay que sumar el coste del tiempo de adaptación necesario al puesto de trabajo del nuevo empleado.

d) La pérdida de los trabajadores más brillantes (tanto los que son objeto de mobbing como aquellos que tienen características parecidas a la víctima y piensan que serán los siguientes).

e) La desaparición de la eficiencia por la extensión de la mediocridad técnica y humana.

f) Un mal clima de trabajo lo que dificultará el trabajo en equipo así como las colaboraciones y la comunicación entre miembros de la organización.

g) Un peor trato tanto a los clientes como a los proveedores (con lo cual disminuirá su número).

Todo ello terminará reflejándose tanto en el producto final que ofrece la empresa como en su cuenta de resultados, acarreando efectos negativos sobre su imagen y reputación.

Tampoco hay que olvidar que las reclamaciones y denuncias interpuestas por la víctima incrementarán el nivel de costes soportados por las empresas al tener que indemnizarlas por los daños inflingidos. Al hacerse público el caso de mobbing se dará una imagen a los clientes, proveedores... y a la sociedad, en general. Una imagen que no resulta positiva, especialmente, en los tiempos actuales en los que existe un mayor conocimiento sobre el mobbing y los efectos destructivos que tiene sobre la persona que lo padece.

Si una actuación de mobbing supone la quiebra del sistema productivo de la empresa, se debe eliminar el origen de la agresión, so pena de acabar destruyendo todo el entramado de la misma, lo que redundaría en la pérdida de la competitividad. "Por ello es imprescindible que el acosador modifique su actitud o desaparezca de la empresa porque el riesgo de extender dicha actuación es muy grande" (Rodríguez, 2004: 201). Que un acosador sea capaz de modificar 
su actitud y sobre todo no volver a actuar de forma tan perversa nunca más sería realmente ideal, pero dado el perfil que presentan este tipo de personas no es muy probable, así que lo más seguro para la empresa es que no continúe trabajando en ella. Ahora bien, si resulta que el dueño de la empresa es el acosador ¿cómo resolver el problema? Desde el punto de vista de que la salud es muy valiosa y no debe ponerse en peligro, la respuesta es: abandonar la empresa, pero haciendo público el motivo del abandono aunque solo sea a nivel de familiares y amigos, el boca a boca funciona, y el acosador no habrá ganado la partida.

La empresa debe ser consciente de que tiene que proteger a sus trabajadores del acoso psicológico en el trabajo aunque solo sea por su propio interés, para conseguir que su cifra de resultados no descienda y no se ponga en peligro su supervivencia.

\subsection{RESPONSABILIDADES DE LA EMPRESA}

Las organizaciones de nuestros días son conscientes de que si realizan una evaluación de la violencia psicológica que existe en su seno la van a encontrar por todas partes. Por ello, es mejor no evaluar, no medir, y eso, a pesar de que hace mucho tiempo que se sabe que en el mundo de la empresa y de los recursos humanos todo aquello que no se mide no puede gestionarse. Pero lo cierto es que la empresa en la que acontece un acto de mobbing tiene un problema que no puede obviar, tiene que resolverlo y lo tiene que hacer lo antes posible. Acabar con este tipo de prácticas exige situar el problema en el contexto correcto que es la asombrosa despreocupación que actualmente presentan muchas organizaciones por sus trabajadores, por sus personas. Por tanto, parece obvio que si se quiere erradicar del mundo laboral el acoso psicológico en el trabajo se necesita un comportamiento serio, ético y responsable por parte de la organización y sus miembros ante este tipo de acoso. Los dirigentes de las empresas tienen que encargarse de muchas cuestiones (administrativas, contables, gestión de personal...) pero eso: 
No les exonera de la obligación de luchar contra la violencia bajo todas sus formas tomando las medidas necesarias para sancionarlas. [...]. Las jerarquias [de las empresas] deberian dejar de defender sistemáticamente, por espiritu corporativo, a los ejecutivos acusados de acoso moral. [...] Ese proceder es lo que les lleva a perpetuarlas $y$ agravarlas. (Hirigoyen, 2001: 269).

La empresa debería preocuparse tanto por las personas como lo hace por las cifras; no dejar actuar a los acosadores y tomar medidas al respecto (Hirigoyen, 2001: 274). La protección de la salud, la seguridad, el respeto y la dignidad de los trabajadores es el elemento central de la responsabilidad de los empresarios en una sociedad democrática (Einarsen y Hauge, 2006: 267).

La mayor o menor importancia que puedan tener los costes económicos derivados del mobbing en la empresa, no es justificación para que la misma decida afrontar el problema solo por este motivo (aunque dicho motivo podría "admitirse por válido" si con ello se consigue erradicar el mobbing), sino que el criterio fundamental que debe seguir la empresa al solucionar este problema debe ser buscar la salud de sus trabajadores y velar para que esta se mantenga en el tiempo. Un empresario responsable tiene que defender los intereses de sus trabajadores y establecer los mecanismos adecuados para conseguir tener y mantener un ambiente de trabajo seguro, apropiado y libre de hostilidades y cualquier otro riesgo. Por otro lado, el no hacerlo le puede costar minar la reputación de la empresa y, tal vez, lo que más le preocupe: su nivel de beneficio al final del ejercicio económico. Piñuel (2001: 34) comenta, dirigiéndose directamente al empresario que si la razón que le mueve para tomar medidas que erradiquen el mobbing en su empresa no es la ética empresarial entonces que al menos lo haga por mantener cifras positivas en su cuenta de resultados, pero va más allá con el siguiente comentario:

Créame si le digo que una cuarta parte de sus trabajadores han experimentado (o experimentarán) en algún momento de sus vidas este triste fenómeno. Puede que usted también... Quién sabe... 
La organización tiene la obligación legal de velar por la salud de sus trabajadores, por tanto, resulta lógico derivar una responsabilidad por los hechos que se han cometido en su seno (Piñuel y Oñate, 2003: 6). Leymann (1996b: 179) afirma que un buen directivo debe ser capaz de leer los primeros síntomas de un proceso de mobbing en curso. Con lo cual ya está haciendo responsable a la organización de este tipo de violencia.

Toda empresa debería poner en funcionamiento una serie de mecanismos para prevenir que en su seno se produzcan casos de mobbing y en el caso de detectar que existen solucionarlos lo más rápido posible. En este proceso de erradicación del mobbing en las organizaciones se podría señalar una serie de pasos a seguir:

1) Adoptar medidas de prevención del mobbing, al mismo tiempo, que se realiza una vigilancia continua a fin de detectar posibles casos de acoso psicológico en el trabajo.

2) En el caso de detectar casos de mobbing, actuar lo más rápido posible, de forma eficaz y con medidas que desalienten la aparición de futuros casos.

Todo lo enunciado es partiendo de la hipótesis de que realmente la empresa quiere que no exista mobbing en su seno, pero si no se cumple tal hipótesis las leyes deben ayudar a la víctima a liberarse de su acosador.

Es cierto que no puede dejarse en manos de los legisladores todos los problemas; la empresa tiene que intentar solucionar por sí misma sus problemas, tiene que asumir responsabilidades y, en este caso que nos ocupa, tener tolerancia cero con el mobbing. No obstante, también la legislación debe hacer un esfuerzo por recoger legalmente este grave problema laboral de nuestro tiempo.

\section{Primero: Adoptar medidas de prevención del mobbing, al mismo tiempo, que se realiza una vigilancia continua a fin de detectar posibles casos de acoso psicológico en el trabajo.}


La empresa para conseguir librarse de la plaga del mobbing debe establecer reglamentos internos y políticas adecuadas sobre el ambiente del trabajo, ética y trato digno e igualitario entre todos los miembros de la organización. Estas normas proporcionarán a los empleadores, a los trabajadores y a sus representantes una guía para identificar, prevenir y resolver los problemas de acoso que se presenten en el lugar de trabajo; lejos de limitarse a sancionar estos comportamientos una vez producidos, tratan, por el contrario, de ofrecer un enfoque preventivo para evitar que estos puedan tener lugar, detectando el problema en sus inicios, cuando la solución es más plausible (Velasco, 2010: 73) y la víctima no ha sufrido muchísimo.

Estos reglamentos deben recoger dos grandes bloques de medidas:

1. Impedir que se produzcan casos de mobbing.

2. En el caso de que sean descubiertos casos de mobbing, establecer medidas urgentes para eliminarlo, indemnizando a la víctima y sancionando al acosador.

Autores como Hirigoyen (2001: 268) consideran que con medidas preventivas

No se podrá en modo alguno cambiar la voluntad de causar daño de un perverso narcisista, pero al menos se le podrá contener y se podrá poner limites a su destructividad. En cuanto a los asalariados que tienden a seguir al grupo, correrán menos riesgo de resbalar si el grupo funciona sanamente.

En el contenido de estos reglamentos y políticas se deben abordar cuestiones tales como las planteadas por Hirigoyen (2001), Rodríguez (2004), Slin (2006) o Velasco (2010):

a) Fomento del compromiso de la empresa hacia objetivos en los que se rechaza la violencia y además sancionarla si se produce, sin mirar quién es la víctima y quién el acosador. 
b) Educar a los trabajadores en normas de buena conducta, definiendo con claridad qué se acepta y qué no se acepta.

c) Formación del personal en la detección de riesgos en el entorno de trabajo y en las estrategias más apropiadas a seguir para hacerles frente.

d) Formación de los trabajadores de cualquier nivel en la resolución de conflictos.

e) Indicar explícitamente el procedimiento a seguir por el trabajador que se considere víctima de mobbing (cómo actuar, a quién acudir...). El procedimiento debe ser simple, confidencial y ofrecer varias alternativas.

f) Desarrollo de reglas claras, explícitas y públicas sobre la resolución de conflictos, estableciendo además con claridad y de forma explícita las sanciones que podrán ser aplicadas al acosador.

g) Instauración de programas de asistencia a los trabajadores con el fin de proporcionar asesoramiento y apoyo a las víctimas.

h) Establecer medidas de seguimiento de la situación una vez se haya resuelto el problema de mobbing e impuestas las sanciones pertinentes.

i) Proporcionar a los trabajadores información clara y concreta de las actividades que deben realizar, cómo realizarlas, medios de los que disponen, objetivos a alcanzar....

j) Diseño, rediseño y control de los procedimientos de trabajo contando con la opinión y experiencia de los trabajadores que los desempeñan, con la finalidad clara y explícita de mejorarlos.

k) Fomento de estilos de dirección más participativos y respetuosos, que permitan un margen significativo de autonomía a los trabajadores y respeten su dignidad como ser humano. 
1) Formación de los directivos y mandos intermedios en liderazgo, dirección de personas, resolución de conflictos, comunicación, habilidades sociales, desarrollo de recursos humanos, prevención del estrés....

m) Establecimiento de sistemas de comunicación eficaces en la organización a todos los niveles.

n) Establecimiento de buenas condiciones de trabajo (prevenir el enrarecimiento del clima laboral, incentivar la colaboración, cooperación y confianza entre los trabajadores, velar por la prevención del estrés en los trabajadores, incentivar las transferencias de conocimiento entre ellos, no la retención de la información, fomentar la formación del trabajador, reducir la precarización e inseguridad laboral...).

o) Establecer sistemas de promoción no perversos, que estén basados en el mérito del trabajador y no en la política, el amiguismo, el nepotismo o el favoritismo.

p) Planificación, diseño y fomento de las relaciones sociales en la empresa. También, sería recomendable que a la hora de seleccionar al personal se tuvieran en cuenta las características que definen el perfil de un acosador, a fin de evitar que este tipo de personas entren en la empresa por muy competentes que parezcan.

Estos reglamentos deben darse a conocer a todos los miembros de la organización, pero la organización ha de ser consciente que aunque hallan reglamentos debe existir en la empresa una vigilancia activa para detectar y eliminar los casos de mobbing, no basta con la existencia de normas escritas es necesario un proceso de vigilancia activa si se quiere erradicar el problema del mobbing.

Estos reglamentos y políticas deben ser elaborados por la dirección de la empresa contando con la participación de los trabajadores y sus representantes, y deben recoger claramente que en la empresa no se aceptará ningún tipo de comportamiento que suponga $\mathrm{o}$ 
pueda inducir a los miembros de la organización a cualquier tipo de acoso. Para lograr que tales reglamentos y políticas sean eficaces se necesita el apoyo de la dirección y el compromiso de todos los miembros de la organización, además los trabajadores necesitan sentir que tales políticas y reglamentos son útiles para prevenir el mobbing y que se aplicarán sea quién sea la víctima y el acosador.

El camino hacia un lugar de trabajo libre de acoso es también el camino hacia unas condiciones de vida y trabajo saludables, igual que en el caso de los riesgos laborales la prevención es la orientación básica para evitar situaciones de acoso psicológico en el trabajo (Aramburu-Zabala, 2002: 348).

La Agencia Europea para la Seguridad y Salud en el Trabajo (2002) ha propuesto una serie de recomendaciones generales para prevenir el mobbing, considerando su prevención como un elemento básico para mejorar la vida laboral y evitar la exclusión social, teniendo en cuenta la adopción de medidas en una fase temprana para evitar un entorno de trabajo destructivo, por lo que las empresas no deberían esperar a recibir las quejas de las víctimas. La Agencia Europea considera necesaria una implicación madura, responsable y éticamente comprometida de las empresas y de los trabajadores y trabajadoras.

Las propuestas de la Agencia Europea son:

a) Ofrecer a cada trabajador o trabajadora la posibilidad de escoger la manera de realizar su trabajo.

b) Reducir el volumen de trabajos monótonos y repetitivos.

c) Aumentar la información sobre objetivos.

d) Desarrollar el estilo democrático de dirección.

e) Evitar especificaciones poco claras de funciones y tareas.

La Agencia además hace una serie de recomendaciones para crear una cultura organizativa con normas y valores contra el acoso psicológico tales como: favorecer la difusión del significado de acoso 
laboral, investigar el alcance y naturaleza del problema de acoso laboral, formular directrices claras para favorecer interacciones sociales positivas...

No obstante todo lo dicho, la mejor forma de convencer a los directivos de las empresas de que las estrategias de prevención son una buena inversión es demostrarles que la existencia del mobbing en sus organizaciones "le sale caro" (Hirigoyen, 2001: 275).

En el estudio empírico realizado por Slin (2006: 421-422, 426-427) se obtuvieron conclusiones muy interesantes. En dicho estudio se muestra cómo, de forma mayoritaria, las organizaciones (tanto públicas como privadas) no facilitaban información alguna sobre el mobbing (lo cual puede llevar a pensar que no tengan reglamentos y políticas antimobbing), además el tema del mobbing no parece tener demasiada prioridad en la agenda de las empresas (estas ante el mobbing mantienen una estrategia reactiva más que preactiva). A lo que se añade que, más de la mitad de los trabajadores creían que sus respectivas compañías no tenían las suficientes capacidades para lidiar con una situación de acoso, en el caso de que se produjera.

Los datos del Barómetro CISNEROS XI (2009) también son muy reveladores ${ }^{7}$, ya que muestran que la mayoría de las organizaciones $(53 \%)$ no disponen de protocolos específicos de actuación ante el mobbing y de aquellas que los tienen solo un escaso 9,6\% cuentan con protocolos eficaces de protección, siendo la tendencia más común entre las empresas que cuentan con protocolos que no los apliquen (20\%). En este sentido, cabe la afirmación de Vélez (2011: 1) de que para eliminar el acoso psicológico en el trabajo "no basta con no cometerlo es necesario no permitirlo".

7 El Barómetro CISNEROS (Cuestionario Individual sobre PSicoterror, Negación, Estigmatización y Rechazo en Organizaciones Sociales) está integrado por una serie de escalas cuyo objetivo es sondear de manera periódica el estado y las consecuencias de la violencia en el entorno laboral de las organizaciones. Y es la primera herramienta que se utilizó para medir la incidencia del fenómeno del acoso psicológico en el trabajo en España. 


\section{Segundo: En el caso de detectar casos de mobbing, actuar lo más rápido posible, de forma eficaz y con medidas que desalienten la aparición de futuros casos.}

Si surgen casos de mobbing en la empresa a pesar de tener protocolos y políticas de prevención (por tener tales medidas no necesariamente se erradica total y definitivamente el mobbing), estos deben ser analizados nuevamente a la luz de los casos que aparezcan. Su análisis ayudará a mejorar tales medidas de prevención, lo recomendable es aprender de ellos para llegar a crear medidas de prevención mucho más eficaces para la organización.

La detección temprana del mobbing y la rapidez en darle una solución correcta evitará un gran sufrimiento a la víctima y podrá servir también como señal de que en la empresa este tipo de prácticas no son permitidas sino todo lo contrario son perseguidas y sancionadas.

El acosador debe ser sancionado públicamente por su delito, no de forma oculta y privada. Y si continúa en la empresa, ha de ser sometido a vigilancia para evitar que vuelva a hacerlo otra vez. Cuanto mayor sea el número de personas que conozcan lo que ha sido capaz de hacer el acosador mucho mejor para el resto de trabajadores de su organización y de las demás. Hacer público lo que es no supone una vulneración de sus derechos, sino todo lo contrario es una forma de defender los derechos del resto.

La víctima debe ser "indemnizada”, pero aquí surge un problema de difícil solución: ¿cómo indemnizar a una persona que ha sufrido acoso psicológico en su puesto de trabajo, que ha intentado ser destruida psicológicamente por el simple capricho de un psicópata y perverso acosador? ¿Puede existir una indemnización adecuada, correcta y justa? Muchas víctimas afirmarían que no, que no la hay (tal vez después de que hayan pasado muchos años y hayan "superado" el sufrimiento pasado, algunas puedan decir cuál puede ser esa "justa indemnización” que merecen). Volver a vivir, sin miedo y con la felicidad en el alma y en el corazón, quizás una víctima de mobbing aceptaría esto como indemnización. 
Tampoco hay que olvidar que una persona cuya reputación profesional e incluso personal ha sido destruida (en este caso por el acosador y sus cómplices), tendrá dificultades para recuperarla; la reputación una vez perdida no es tan fácil de restituir, de recuperar.

La empresa cumpliendo con las responsabilidades que deben asumir en los casos de mobbing que se hayan producido en su seno debería proporcionar a la víctima, de forma gratuita, ayuda del tipo que precise para que recupere la salud perdida. De esta forma la víctima no tendría tantos problemas económicos por tener que hacer frente a los costes de la ayuda especializada que necesita. En este sentido, se pronuncia también Leymann (1996b: 179), al afirmar que conforme un proceso de acoso se desarrolla es obligación de la dirección de la empresa proteger al individuo en peligro. Se debe prevenir su estigmatización y debe ser capaz de recuperar su reputación y habilidades previas. Se le debe sugerir que tome baja por enfermedad y ofrecerle la posibilidad de reintegración profesional. Por el contrario, permitir la desgracia de una persona a través de un proceso de acosos, y por ello, desprenderse de ella, deberá ser considerado, por lo menos, como un fracaso de gestión mayúsculo.

En el estudio realizado por Slin (2006: 423), citado anteriormente, los resultados obtenidos muestran que la mayoría de las víctimas se muestran bastante decepcionadas en cuanto al apoyo y la ayuda recibida por sus superiores y por la organización; muchos afirmaron no haber recibido ningún tipo de ayuda de la dirección a pesar de haberla solicitado. Resultados similares aporta el Barómetro CISNEROS XI (2009) cuando emite:

a) 2 de cada 3 víctimas sienten que son abandonadas por la empresa.

b) Ante los casos de mobbing la empresa presenta una gran pasividad e inhibición. 
En cuanto a las personas que dentro de la organización asuman las tareas de prevención, vigilancia y detección y solución de los casos de mobbing, estas deberían poseer amplios conocimientos sobre el tema del mobbing además de conocer la empresa (funcionamiento, normas implícitas e explícitas, estructura de poder...). Es preciso poseer conocimientos sobre el problema y sobre el lugar donde acontece para encontrar la solución acertada.

Todo lo anterior parte de la base de que la empresa por si misma sea capaz de dar una solución acertada al caso del mobbing. Rojas (2005: 144) considera que ante un posible caso de mobbing detectado en la empresa no parece que deba dejarse la investigación, exclusivamente en manos de la empresa, especialmente, si hay sospechas de una fuerte implicación en la posible agresión de la propia dirección de la misma. Así, considera que sería conveniente regular un procedimiento especial que obligue a la apertura de un expediente de investigación en el que se nombre un instructor, con presencia siempre de los sindicados o de los representantes legales de los trabajadores. Estos como organización representativa y de defensa de los trabajadores tienen un papel fundamental en la lucha contra el mobbing, utilizando para ello la cercanía que tienen con el trabajador, allí donde se le está haciendo el daño, es decir, en su puesto de trabajo. Para que los sindicatos puedan cumplir esta importante labor es requisito necesario que dichos representantes conozcan qué es el mobbing, cuáles son sus consecuencias, etc., ello le permitirá detectar los casos de mobbing e iniciar una intervención sindical inmediata.

\section{Conclusiones}

El mobbing se ha considerado como la plaga laboral del siglo XXI, ocasionador de graves daños para la víctima que lo sufre, para la empresa en la que se produce e incluso puede afirmarse que perjudica a la sociedad en general. Este tipo de acoso tiene lugar en el puesto de trabajo, dentro de una organización, por tanto esta sufre 
sus consecuencias pero también debe asumir su responsabilidad e intentar erradicarlo. La empresa debe velar por sus trabajadores, pues constituyen su capital más valioso, ella es responsable de lo que les acontezca cuando están en su seno. Así, un problema tan destructivo para el ser humano (y también para la empresa) como es el mobbing está obligada a resolverlo: es más debería no tener que llegar a resolver ningún caso de mobbing, pues ello significaría que ha tomado todas las medidas necesarias a priori para prevenir tales acosos, que ha cumplido de forma correcta con sus obligaciones hacia sus empleados estableciendo todos los mecanismos necesarios para prevenir tales situaciones.

Para erradicar el mobbing es necesario querer hacerlo y hacerlo, para eso se debe creer en que dicha conducta es una plaga que debe desaparecer, por lo que la empresa no puede negar su responsabilidad en este proceso de erradicación.

\section{Referencias bibliográficas}

Agencia Europea para la Seguridad y Salud en el Trabajo (2002). Acoso moral en el trabajo. [Hoja informativa 23]. Bruselas: Agencia Europea para la Seguridad y Salud en el Trabajo.

Aramburu-Zabala Higuera, L. (2002). Respuesta al acoso laboral. Programas y estrategia. Cuadernos de Relaciones Laborales, 20(29), 337-350.

Asociaciones Profesionales de los Cuerpos Técnicos del Ministerio de Hacienda. (2009). Barómetro CISNEROS XI. Madrid: Universidad de Alcalá de Henares.

Einarsen, S., \& Hauge, L. J. (2006). Antecedentes y consecuencias del acoso psicológico en el trabajo: una revisión de la literatura. Revista de Psicología del Trabajo y de las Organizaciones, 22(3), 251-273. 
Fundación Europea para la mejora de las Condiciones de Vida y de Trabajo (2007). Cuarta encuesta europea sobre las condiciones de vida y de trabajo. Luxemburgo: Oficina de publicaciones oficiales de la Unión Europea.

Fundación Europea para la mejora de las Condiciones de Vida y de Trabajo (2012). Quinta encuesta europea sobre las condiciones de vida y de trabajo. Luxemburgo: Oficina de Publicaciones Oficiales de la Unión Europea.

Girard, R. (2002). Veo a Satán caer como el relámpago. Barcelona: Anagrama.

Hirigoyen, M. F. (2001). El acoso moral en el trabajo. Distinguir lo verdadero de lo falso. Barcelona: Ediciones Paidós Ibérica.

Leymann, H. (1996a). Mobbing : La persécution au travail. París: Editorial Du Seuil.

Leymann, H. (1996b). Contenido y desarrollo del acoso grupal/moral ("mobbing") en el trabajo. European Journal of Work and Organizational Psychology, 5(2), 165-184.

López Cabarcos, M. A., \& Vázquez Rodríguez, P. (2003). Mobbing. Cómo prevenir, identificar y solucionar el acoso psicológico en el trabajo. Madrid: Editorial Pirámide.

Moreno Jiménez, B., Rodríguez Muñoz, A., Garrosa Hernández, E. y Morante Benadero, M. E. (2005). Antecedentes organizacionales del acoso psicológico en el trabajo: Un estudio exploratorio. Psicothema, 17(4), 627-632.

Muñoz Floresi, H., Guerra de los Santos, J. M., Barón Duque, M., \& Munduate Jaca, L. (2006). El acoso psicológico desde una perspectiva organizacional. Papel del clima organizacional y los procesos de cambio. Revista de Psicología del Trabajo y de las Organizaciones, 22(3), 347-361. 
Piñuel y Zabala, I. (2001). Mobbing: Cómo sobrevivir al acoso psicológico en el trabajo. Santander: Sal Terrae.

Piñuel y Zabala, I. (2004). Neomanagement. Jefes tóxicos y sus víctimas. Madrid: Aguilar.

Piñuel y Zabala, I. \& Oñate Cantero, A. (2003). El mobbing o acoso psicológico en el trabajo en España. Congreso Internacional Virtual: Intangibles e Interdisciplinariedad (pp. 1-20). Madrid: Instituto de Innovación Educativa y Desarrollo Directivo.

Real Academia Española y Asociación de Academias de la Lengua Española (2005). Diccionario panhispánico de dudas. Madrid: Santillana.

Rodríguez López, P. (2004). El acoso psicológico en el trabajo. La responsabilidad en el acoso moral en el trabajo. Madrid: Ediciones Jurídicas Dijusa.

Rodríguez Muñoz, A., Martínez Gamarra, M., Moreno Jiménez, B., \& Gálvez Herrer, M. (2006). Predictores organizacionales del acoso psicológico en el trabajo: Aplicación del modelo de demandas y recursos laborales. Revista de Psicología del Trabajo $y$ de las Organizaciones, 22(3), 333-345.

Rojas Rivero, G. P. (2005). Delimitación, prevención y tutela del acoso laboral. Albacete: Editorial Bomarzo.

Sánchez Cabaco, A. (1999). Variables individuales (cognitivoemocionales) y grupales en las nuevas patologías: El caso del mobbing o acoso psicológico en las organizaciones. Revista Iberoamericana de Educación, Saludy Trabajo, N. ${ }^{\circ}$ 0, 235-250.

Slin, D. (2006). ¿Se preocupan las organizaciones por el acoso psicológico en el trabajo? Percepciones de los trabajadores sobre las medidas organizacionales contra el acoso psicológico. Revista de Psicologia del Trabajo y de las Organizaciones, $22(3)$ 413-431. 
Topa Cantisano, G., Morales Domínguez, J. F., \& Gallastegui Galán, J. A. (2006). Acoso laboral: relaciones con la cultura organizacional y los resultados personales", Psicothema, 18(4), 766-771.

Vega, G., \& Comer, D. (2005). Sticks and stones may break your bones but words can break your spirit: bullying in the workplace. Journal of Business Ethics, 58, 101-109.

Velasco Portero, M. T. (2010). Mobbing, acoso laboral y acoso por razón de sexo. Guia para la empresa y las personas trabajadoras. Madrid: Tecnos.

Vélez Rodríguez, L. M. (2011). Mobbing: conserjería y consultaría en Puerto Rico. Revista Jurídica de LexJuris de Puerto Rico, 16(1), 1-10.

\section{María Concepción Verona Martel}

Es licenciada en Ciencias Económicas y Empresariales, Universidad de Las Palmas de Gran Canaria, España; PH.D. en Ciencias Económicas y Empresariales, Universidad de Las Palmas de Gran Canaria, España; profesora titular de la Facultad de Economía, Empresa y Turismo, de la Universidad de Las Palmas de Gran Canaria, España; miembro del Grupo de Investigación Información Contable, Economía Financiera y Desarrollo Sostenible (INFISOC), Universidad de Las Palmas de Gran Canaria.

E-mail:cverona@defc.ulpgc.es. 


\section{José Juan Déniz Mayor}

Es licenciado en Ciencias Económicas y Empresariales, Universidad de Las Palmas de Gran Canaria, España; con doctorado en Ciencias Económicas y Empresariales por la Universidad de Las Palmas de Gran Canaria, España; es profesor titular de la Facultad de Economía, Empresa y Turismo, de la Universidad de Las Palmas de Gran Canaria; miembro del Grupo de Investigación Información Contable, Economía Financiera y Desarrollo Sostenible (INFISOC), Universidad de Las Palmas de Gran Canaria.

E-mail: josejuan.deniz@ulpgc.es

\section{Raquel Santana Mateo}

Graduada en Turismo por la Universidad de Las Palmas de Gran Canaria.

E-mail: rakel15700@hotmail.com

Recibido: 22/03/2014 Aprobado: 09/08/2014 